\title{
Algebraic Numbers
}

\author{
Yasushige Watase \\ Suginami-ku Matsunoki 6 \\ 3-21 Tokyo, Japan
}

\begin{abstract}
Summary. This article provides definitions and examples upon an integral element of unital commutative rings. An algebraic number is also treated as consequence of a concept of "integral". Definitions for an integral closure, an algebraic integer and a transcendental numbers [14, 1, 10 and [7 are included as well. As an application of an algebraic number, this article includes a formal proof of a ring extension of rational number field $\mathbb{Q}$ induced by substitution of an algebraic number to the polynomial ring of $\mathbb{Q}[x]$ turns to be a field.
\end{abstract}

\section{MSC: 11R04 13B21 03B35}

Keywords: algebraic number; integral dependency

MML identifier: ALGNUM_1, version: 8.1.05 5.39.1282

\section{Preliminaries}

From now on $i, j$ denote natural numbers and $A, B$ denote rings.

Now we state the propositions:

(1) Let us consider rings $L_{1}, L_{2}, L_{3}$. Suppose $L_{1}$ is a subring of $L_{2}$ and $L_{2}$ is a subring of $L_{3}$. Then $L_{1}$ is a subring of $L_{3}$.

(2) $\quad \mathbb{F}_{\mathbb{Q}}$ is a subfield of $\mathbb{C}_{\mathrm{F}}$.

(3) $\quad \mathbb{F}_{\mathbb{Q}}$ is a subring of $\mathbb{C}_{\mathrm{F}}$.

(4) $\mathbb{Z}^{\mathrm{R}}$ is a subring of $\mathbb{C}_{\mathrm{F}}$.

Let us consider elements $x, y$ of $B$ and elements $x_{1}, y_{1}$ of $A$. Now we state the propositions:

(5) If $A$ is a subring of $B$ and $x=x_{1}$ and $y=y_{1}$, then $x+y=x_{1}+y_{1}$.

(6) If $A$ is a subring of $B$ and $x=x_{1}$ and $y=y_{1}$, then $x \cdot y=x_{1} \cdot y_{1}$.

Let $c$ be a complex. Observe that $c\left(\in \mathbb{C}_{\mathrm{F}}\right)$ reduces to $c$. 


\section{Extended Evaluation Function}

Let $A, B$ be rings, $p$ be a polynomial over $A$, and $x$ be an element of $B$. The functor $\operatorname{ExtEval}(p, x)$ yielding an element of $B$ is defined by

(Def. 1) there exists a finite sequence $F$ of elements of $B$ such that $i t=\sum F$ and len $F=\operatorname{len} p$ and for every element $n$ of $\mathbb{N}$ such that $n \in \operatorname{dom} F$ holds $F(n)=p\left(n-^{\prime} 1\right)(\in B) \cdot \operatorname{power}_{B}\left(x, n-^{\prime} 1\right)$.

Now we state the proposition:

(7) Let us consider an element $n$ of $\mathbb{N}$, rings $A, B$, and an element $z$ of $A$. Suppose $A$ is a subring of $B$. Then $\operatorname{power}_{B}(z(\in B), n)=\operatorname{power}_{A}(z, n)(\in$ $B)$. The theorem is a consequence of (6).

Let us consider elements $x_{1}, x_{2}$ of $A$. Now we state the propositions:

(8) If $A$ is a subring of $B$, then $x_{1}(\in B)+x_{2}(\in B)=\left(x_{1}+x_{2}\right)(\in B)$. The theorem is a consequence of $(5)$.

(9) If $A$ is a subring of $B$, then $x_{1}(\in B) \cdot x_{2}(\in B)=\left(x_{1} \cdot x_{2}\right)(\in B)$. The theorem is a consequence of (6).

(10) Let us consider a finite sequence $F$ of elements of $A$, and a finite sequence $G$ of elements of $B$. If $A$ is a subring of $B$ and $F=G$, then $\left(\sum F\right)(\in B)=$ $\sum G$.

Proof: Define $\mathcal{P}$ [natural number] $\equiv$ for every finite sequence $F$ of elements of $A$ for every finite sequence $G$ of elements of $B$ such that len $F=\$_{1}$ and $F=G$ holds $\left(\sum F\right)(\in B)=\sum G$. $\mathcal{P}[0]$ by [13, (43)]. For every natural number $n$ such that $\mathcal{P}[n]$ holds $\mathcal{P}[n+1]$ by [4, (4)], [5, (3)], [4, (59)], [3, (11)]. For every natural number $n, \mathcal{P}[n]$ from [3, Sch. 2].

(11) Let us consider a natural number $n$, an element $x$ of $A$, and a polynomial $p$ over $A$. Suppose $A$ is a subring of $B$. Then $p\left(n-^{\prime} 1\right)(\in B) \cdot \operatorname{power}_{B}(x(\in$ $\left.B), n-^{\prime} 1\right)=\left(p\left(n-^{\prime} 1\right) \cdot \operatorname{power}_{A}\left(x, n-^{\prime} 1\right)\right)(\in B)$. The theorem is a consequence of (9) and (7).

(12) Let us consider an element $x$ of $A$, and a polynomial $p$ over $A$. Suppose $A$ is a subring of $B$. Then $\operatorname{ExtEval}(p, x(\in B))=(\operatorname{eval}(p, x))(\in B)$.

Proof: Consider $F_{1}$ being a finite sequence of elements of $B$ such that $\operatorname{ExtEval}(p, x(\in B))=\sum F_{1}$ and len $F_{1}=\operatorname{len} p$ and for every element $n$ of $\mathbb{N}$ such that $n \in \operatorname{dom} F_{1}$ holds $F_{1}(n)=p\left(n-^{\prime} 1\right)(\in B) \cdot$ power $_{B}(x(\in$ $\left.B), n-{ }^{\prime} 1\right)$. Consider $F_{2}$ being a finite sequence of elements of $A$ such that $\operatorname{eval}(p, x)=\sum F_{2}$ and len $F_{2}=\operatorname{len} p$ and for every element $n$ of $\mathbb{N}$ such that $n \in \operatorname{dom} F_{2}$ holds $F_{2}(n)=p\left(n-{ }^{\prime} 1\right) \cdot \operatorname{power}_{A}\left(x, n-^{\prime} 1\right) . F_{1}=F_{2}$ by [12, (29)], [5, (3)], (19).

(13) Let us consider an element $x$ of $B$. Then $\operatorname{ExtEval}(\mathbf{0} . A, x)=0_{B}$. 
(14) Let us consider non degenerated rings $A, B$, and an element $x$ of $B$. If $A$ is a subring of $B$, then $\operatorname{ExtEval}(\mathbf{1} . A, x)=1_{B}$.

(15) Let us consider an element $x$ of $B$, and polynomials $p, q$ over $A$. Suppose $A$ is a subring of $B$. Then $\operatorname{ExtEval}(p+q, x)=\operatorname{ExtEval}(p, x)+\operatorname{ExtEval}(q, x)$. The theorem is a consequence of (8).

(16) Let us consider polynomials $p, q$ over $A$. Suppose $A$ is a subring of $B$ and len $p>0$ and len $q>0$. Let us consider an element $x$ of $B$. Then ExtEval(Leading-Monomial $p *$ Leading-Monomial $q, x)=\left(p\left(\operatorname{len} p-{ }^{\prime} 1\right)\right.$. $\left.q\left(\operatorname{len} q-^{\prime} 1\right)\right)(\in B) \cdot \operatorname{power}_{B}\left(x, \operatorname{len} p+\operatorname{len} q-^{\prime} 2\right)$. The theorem is a consequence of (13).

(17) Let us consider a polynomial $p$ over $A$, and an element $x$ of $B$. Suppose $A$ is a subring of $B$. Then ExtEval(Leading-Monomial $p, x)=p\left(\operatorname{len} p-^{\prime} 1\right)(\in$ $B) \cdot \operatorname{power}_{B}\left(x\right.$, len $\left.p-{ }^{\prime} 1\right)$. The theorem is a consequence of (13).

Let us consider a commutative ring $B$, polynomials $p, q$ over $A$, and an element $x$ of $B$. Now we state the propositions:

(18) Suppose $A$ is a subring of $B$. Then ExtEval(Leading-Monomial $p *$ LeadingMonomial $q, x)=\operatorname{ExtEval(Leading-Monomial~} p, x) \cdot$ ExtEval(Leading-Monomial $q, x)$. The theorem is a consequence of (16), (9), (17), and (13).

(19) Suppose $A$ is a subring of $B$. Then ExtEval(Leading-Monomial $p * q, x)=$ $\operatorname{ExtEval}($ Leading-Monomial $p, x) \cdot \operatorname{ExtEval}(q, x)$.

Proof: Set $p=$ Leading-Monomial $p_{1}$. Define $\mathcal{P}$ [natural number] $\equiv$ for every polynomial $q$ over $A$ such that len $q=\$_{1} \operatorname{holds} \operatorname{ExtEval}(p * q, x)=$ $\operatorname{ExtEval}(p, x) \cdot \operatorname{ExtEval}(q, x)$. For every natural number $k$ such that for every natural number $n$ such that $n<k$ holds $\mathcal{P}[n]$ holds $\mathcal{P}[k]$ by [9, (16)], [8, (31)], (15), (18). For every natural number $n, \mathcal{P}[n]$ from [3, Sch. 4].

(20) If $A$ is a subring of $B$, then $\operatorname{ExtEval}(p * q, x)=\operatorname{ExtEval}(p, x) \cdot \operatorname{ExtEval}(q, x)$. Proof: Define $\mathcal{P}$ [natural number] $\equiv$ for every polynomial $p$ over $A$ such that len $p=\$_{1} \operatorname{holds} \operatorname{ExtEval}(p * q, x)=\operatorname{ExtEval}(p, x) \cdot \operatorname{ExtEval}(q, x) \cdot$ For every natural number $k$ such that for every natural number $n$ such that $n<k$ holds $\mathcal{P}[n]$ holds $\mathcal{P}[k]$ by [9, (16)], [8, (32)], (15), (19). For every natural number $n, \mathcal{P}[n]$ from [3, Sch. 4].

(21) Let us consider an element $x$ of $B$, and an element $z_{0}$ of $A$. Suppose $A$ is a subring of $B$. Then $\operatorname{ExtEval}\left(\left\langle z_{0}\right\rangle, x\right)=z_{0}(\in B)$. The theorem is a consequence of (13).

(22) Let us consider an element $x$ of $B$, and elements $z_{0}, z_{1}$ of $A$. Suppose $A$ is a subring of $B$. Then $\operatorname{ExtEval}\left(\left\langle z_{0}, z_{1}\right\rangle, x\right)=z_{0}(\in B)+z_{1}(\in B) \cdot x$. The theorem is a consequence of (13). 


\section{Integral Element and Algebraic Numbers}

Let $A, B$ be rings and $x$ be an element of $B$. We say that $x$ is integral over $A$ if and only if

(Def. 2) there exists a polynomial $f$ over $A$ such that LC $f=1_{A}$ and $\operatorname{ExtEval}(f, x)=0_{B}$.

Now we state the proposition:

(23) Let us consider a non degenerated ring $A$, and an element $a$ of $A$. If $A$ is a subring of $B$, then $a(\in B)$ is integral over $A$. The theorem is a consequence of (12).

Let $A$ be a non degenerated ring and $B$ be a ring. Assume $A$ is a subring of $B$. The integral closure over $A$ in $B$ yielding a non empty subset of $B$ is defined by the term

(Def. 3) $\{z$, where $z$ is an element of $B: z$ is integral over $A\}$.

Let $c$ be a complex. We say that $c$ is algebraic if and only if

(Def. 4) there exists an element $x$ of $\mathbb{C}_{\mathrm{F}}$ such that $x=c$ and $x$ is integral over $\mathbb{F}_{\mathbb{Q}}$.

Let $x$ be an element of $\mathbb{C}_{\mathrm{F}}$. Note that $x$ is algebraic if and only if the condition (Def. 5) is satisfied.

(Def. 5) $x$ is integral over $\mathbb{F}_{\mathbb{Q}}$.

Let $c$ be a complex. We say that $c$ is algebraic integer if and only if

(Def. 6) there exists an element $x$ of $\mathbb{C}_{\mathrm{F}}$ such that $x=c$ and $x$ is integral over $\mathbb{Z}^{\mathrm{R}}$.

Let $x$ be an element of $\mathbb{C}_{\mathrm{F}}$. Observe that $x$ is algebraic integer if and only if the condition (Def. 7) is satisfied.

(Def. 7) $x$ is integral over $\mathbb{Z}^{\mathrm{R}}$.

Let $x$ be a complex. We introduce the notation $x$ is transcendental as an antonym for $x$ is algebraic.

Note that every complex which is rational is also algebraic and there exists a complex which is algebraic and there exists an element of $\mathbb{C}_{F}$ which is algebraic and every complex which is integer is also algebraic integer and there exists a complex which is algebraic integer and there exists an element of $\mathbb{C}_{F}$ which is algebraic integer.

Let $A, B$ be rings and $x$ be an element of $B$. The functor $\operatorname{AnnPoly}(x, A)$ yielding a non empty subset of $\operatorname{PolyRing}(A)$ is defined by the term

(Def. 8) $\left\{p\right.$, where $p$ is a polynomial over $\left.A: \operatorname{ExtEval}(p, x)=0_{B}\right\}$.

Now we state the propositions: 
(24) Let us consider rings $A, B$, an element $w$ of $B$, and elements $x, y$ of PolyRing $(A)$. Suppose $A$ is a subring of $B$ and $x, y \in \operatorname{AnnPoly}(w, A)$. Then $x+y \in \operatorname{AnnPoly}(w, A)$. The theorem is a consequence of (15).

(25) Let us consider a commutative ring $B$, an element $z$ of $B$, and elements $p, x$ of PolyRing $(A)$. Suppose $A$ is a subring of $B$ and $x \in \operatorname{AnnPoly}(z, A)$. Then $p \cdot x \in \operatorname{AnnPoly}(z, A)$. The theorem is a consequence of (20).

(26) Let us consider a commutative ring $B$, an element $w$ of $B$, and elements $p, x$ of PolyRing $(A)$. Suppose $A$ is a subring of $B$ and $x \in \operatorname{AnnPoly}(w, A)$. Then $x \cdot p \in \operatorname{AnnPoly}(w, A)$. The theorem is a consequence of (20).

(27) Let us consider a non degenerated ring $A$, a non degenerated commutative ring $B$, and an element $w$ of $B$. Suppose $A$ is a subring of $B$. Then $\operatorname{AnnPoly}(w, A)$ is a proper ideal of $\operatorname{PolyRing}(A)$.

Proof: $\operatorname{AnnPoly}(w, A)$ is closed under addition. $\operatorname{AnnPoly}(w, A)$ is left ideal. $\operatorname{AnnPoly}(w, A)$ is right ideal. $\operatorname{AnnPoly}(w, A)$ is proper by [8, (37)], (14).

\section{Properties of Polynomial Ring over Principal Ideal Domain}

From now on $K, L$ denote fields.

Now we state the propositions:

(28) Let us consider fields $K, L$, and an element $w$ of $L$. Suppose $K$ is a subring of $L$. Then there exists an element $g$ of PolyRing $(K)$ such that $\{g\}$-ideal $=\operatorname{AnnPoly}(w, K)$. The theorem is a consequence of $(27)$.

(29) Let us consider fields $K, L$, and an element $z$ of $L$. Suppose $z$ is integral over $K$. Then $\operatorname{AnnPoly}(z, K) \neq\left\{0_{\text {PolyRing }(K)}\right\}$.

Proof: Consider $f$ being a polynomial over $K$ such that LC $f=1_{K}$ and $\operatorname{ExtEval}(f, z)=0_{L} \cdot f \notin\left\{0_{\text {PolyRing }(K)}\right\}$ by [2, (47), (64)], [11, (7)].

(30) Let us consider a field $K$, and an element $p$ of PolyRing $(K)$. Suppose $p \neq \mathbf{0}$. $K$. Then $p$ is a non zero element of the carrier of PolyRing $(K)$.

Let us consider fields $K, L$ and an element $w$ of $L$. Now we state the propositions:

(31) If $K$ is a subring of $L$, then $\operatorname{AnnPoly}(w, K)$ is quasi-prime. The theorem is a consequence of (20).

(32) If $K$ is a subring of $L$ and $w$ is integral over $K$, then $\operatorname{AnnPoly}(w, K)$ is prime. The theorem is a consequence of (31) and (27).

(33) Let us consider fields $K, L$, and an element $z$ of $L$. Suppose $K$ is a subring of $L$ and $z$ is integral over $K$. Then there exists an element $f$ of PolyRing $(K)$ such that 
(i) $f \neq \mathbf{0} . K$, and

(ii) $\{f\}$-ideal $=\operatorname{AnnPoly}(z, K)$, and

(iii) $f=\operatorname{NormPoly~} f$.

The theorem is a consequence of (28), (29), and (30).

(34) Let us consider fields $K, L$, an element $z$ of $L$, and elements $f, g$ of PolyRing $(K)$. Suppose $z$ is integral over $K$ and $\{f\}$-ideal $=\operatorname{AnnPoly}(z, K)$ and $f=$ NormPoly $f$ and $\{g\}$-ideal $=\operatorname{AnnPoly}(z, K)$ and $g=$ NormPoly $g$. Then $f=g$. The theorem is a consequence of (29) and (30).

Let $K, L$ be fields and $z$ be an element of $L$. Assume $K$ is a subring of $L$ and $z$ is integral over $K$. The minimal polynomial of $z$ over $K$ yielding an element of the carrier of PolyRing $(K)$ is defined by

(Def. 9) $i t \neq \mathbf{0} . K$ and $\{i t\}$-ideal $=\operatorname{AnnPoly}(z, K)$ and $i t=$ NormPoly $i t$.

Assume $K$ is a subring of $L$ and $z$ is integral over $K$. The degree of algebraic number $z$ over $K$ yielding an element of $\mathbb{N}$ is defined by the term

(Def. 10) $\operatorname{deg}($ the minimal polynomial of $z$ over $K$ ).

Let $A, B$ be rings and $x$ be an element of $B$. The functor $\operatorname{HomExtEval}(x, A)$ yielding a function from PolyRing $(A)$ into $B$ is defined by

(Def. 11) for every polynomial $p$ over $A, i t(p)=\operatorname{ExtEval}(p, x)$.

Let $x$ be an element of $\mathbb{C}_{\mathrm{F}}$. Note that $\operatorname{Hom} \operatorname{ExtEval}\left(x, \mathbb{F}_{\mathbb{Q}}\right)$ is unity-preserving, additive, and multiplicative.

Now we state the propositions:

(35) Let us consider an element $x$ of $\mathbb{C}_{\mathrm{F}}$.

Then $\mathbb{C}_{\mathrm{F}}$ is (PolyRing $\left(\mathbb{F}_{\mathbb{Q}}\right)$ )-homomorphic.

(36) Let us consider an element $x$ of $B$, and an object $z$.

If $z \in \operatorname{rng} \operatorname{Hom} \operatorname{ExtEval}(x, A)$, then $z \in B$.

Let $x$ be an element of $\mathbb{C}_{\mathrm{F}}$. The functor $\mathrm{FQ}(x)$ yielding a subset of $\mathbb{C}_{\mathrm{F}}$ is defined by the term

(Def. 12) rng $\operatorname{HomExtEval}\left(x, \mathbb{F}_{\mathbb{Q}}\right)$.

Let us note that $\mathrm{FQ}(x)$ is non empty.

Let us consider elements $x, z_{1}, z_{2}$ of $\mathbb{C}_{\mathrm{F}}$. Now we state the propositions:

(37) If $z_{1}, z_{2} \in \mathrm{FQ}(x)$, then $z_{1}+z_{2} \in \mathrm{FQ}(x)$. The theorem is a consequence of (3) and (15).

(38) If $z_{1}, z_{2} \in \mathrm{FQ}(x)$, then $z_{1} \cdot z_{2} \in \mathrm{FQ}(x)$. The theorem is a consequence of (3) and (20).

(39) Let us consider an element $x$ of $\mathbb{C}_{\mathrm{F}}$, and an element $a$ of $\mathbb{F}_{\mathbb{Q}}$. Then $a \in \mathrm{FQ}(x)$. The theorem is a consequence of (3) and (21). 
Let $x$ be an element of $\mathbb{C}_{\mathrm{F}}$. The functor $\mathrm{FQ}$-add $(x)$ yielding a binary operation on $\mathrm{FQ}(x)$ is defined by the term

(Def. 13) $+{ }_{\mathbb{C}} \nmid \mathrm{FQ}(x)$.

The functor FQ-mult $(x)$ yielding a binary operation on $\mathrm{FQ}(x)$ is defined by the term

(Def. 14) $\cdot \mathbb{C}\lceil\mathrm{FQ}(x)$.

Let us consider an element $x$ of $\mathbb{C}_{\mathrm{F}}$ and elements $z, w$ of $\mathrm{FQ}(x)$. Now we state the propositions:

(40) $(\mathrm{FQ}-\operatorname{add}(x))(z, w)=z+w$.

(41) $\quad(\mathrm{FQ}-\operatorname{mult}(x))(z, w)=z \cdot w$.

(42) Let us consider an element $x$ of $\mathbb{C}_{\mathrm{F}}$. Then $1_{\mathbb{C}_{\mathrm{F}}}(\in \mathrm{FQ}(x))=1_{\mathbb{C}_{\mathrm{F}}}$. The theorem is a consequence of (3) and (39).

(43) $\left(-1_{\mathbb{F}_{\mathbb{Q}}}\right)\left(\in \mathbb{C}_{\mathrm{F}}\right)=-1_{\mathbb{C}_{\mathrm{F}}}$. The theorem is a consequence of (3).

Let $x$ be an element of $\mathbb{C}_{\mathrm{F}}$. The functor $\mathbb{Q}[x]$ yielding a strict, non empty double loop structure is defined by the term

(Def. 15) $\left\langle\mathrm{FQ}(x), \mathrm{FQ}-\operatorname{add}(x), \mathrm{FQ}-\operatorname{mult}(x), 1_{\mathbb{C}_{\mathrm{F}}}(\in \mathrm{FQ}(x)), 0_{\mathbb{C}_{\mathrm{F}}}(\in \mathrm{FQ}(x))\right\rangle$.

Now we state the proposition:

(44) Let us consider an element $x$ of $\mathbb{C}_{\mathrm{F}}$. Then $\mathbb{Q}[x]$ is a ring.

Proof: Reconsider $Z=\left\langle\mathrm{FQ}(x), \mathrm{FQ}-\operatorname{add}(x), \mathrm{FQ}-\operatorname{mult}(x), 1_{\mathbb{C}_{\mathrm{F}}}(\in \mathrm{FQ}(x))\right.$, $\left.0_{\mathbb{C}_{\mathrm{F}}}(\in \mathrm{FQ}(x))\right\rangle$ as a non empty double loop structure. For every elements $v, w$ of $Z, v+w=w+v$. For every elements $u, v, w$ of $Z,(u+v)+w=$ $u+(v+w)$. For every element $v$ of $Z, v+0_{Z}=v$. Every element of $Z$ is right complementable by (36), [6, (9)], (39), (43). For every elements $a, b, v$ of $Z,(a+b) \cdot v=a \cdot v+b \cdot v$. For every elements $a, v, w$ of $Z$, $a \cdot(v+w)=a \cdot v+a \cdot w$ and $(v+w) \cdot a=v \cdot a+w \cdot a$. For every elements $a, b, v$ of $Z,(a \cdot b) \cdot v=a \cdot(b \cdot v)$. For every element $v$ of $Z, v \cdot 1_{Z}=v$ and $1_{Z} \cdot v=v$.

Let $x$ be an element of $\mathbb{C}_{\mathrm{F}}$. One can verify that $\mathbb{Q}[x]$ is Abelian, addassociative, right zeroed, right complementable, associative, well unital, and distributive.

Let $z$ be an element of $\mathbb{C}_{\mathrm{F}}$. One can verify that $\mathbb{Q}[z]$ is integral domain-like, commutative, and non degenerated.

Now we state the proposition:

(45) Let us consider an element $x$ of $\mathbb{C}_{\mathrm{F}}$. Then $\mathbb{Q} \times \mathbb{Q} \subseteq \mathrm{FQ}(x) \times \mathrm{FQ}(x) \subseteq \mathbb{C} \times$ $\mathbb{C}$. The theorem is a consequence of $(39)$.

Let us consider an element $x$ of $\mathbb{C}_{\mathrm{F}}$. Now we state the propositions:

(46) The addition of $\mathbb{F}_{\mathbb{Q}}=$ (the addition of $\left.\mathbb{Q}[x]\right) \uparrow \mathbb{Q}$. The theorem is a consequence of (45). 
(47) The multiplication of $\mathbb{F}_{\mathbb{Q}}=$ (the multiplication of $\left.\mathbb{Q}[x]\right) \uparrow \mathbb{Q}$. The theorem is a consequence of (45).

(48) $\quad \mathbb{F}_{\mathbb{Q}}$ is a subring of $\mathbb{Q}[x]$. The theorem is a consequence of (46), (47), (42), (3), and (39).

Let us consider elements $f, g$ of PolyRing $(K)$. Now we state the propositions:

(49) Suppose $f \neq 0_{\operatorname{PolyRing}(K)}$ and $\{f\}$-ideal is prime and $g \notin\{f\}$-ideal. Then $\{f, g\}$-ideal $=$ the carrier of $\operatorname{PolyRing}(K)$.

(50) Suppose $f \neq 0_{\operatorname{PolyRing}(K)}$ and $\{f\}$-ideal is prime and $g \notin\{f\}$-ideal. Then $\{f\}$-ideal and $\{g\}$-ideal are co-prime. The theorem is a consequence of (49).

(51) Let us consider an element $x$ of $\mathbb{C}_{F}$, and an element $a$ of $\mathbb{Q}[x]$. Then there exists an element $g$ of $\operatorname{PolyRing}\left(\mathbb{F}_{\mathbb{Q}}\right)$ such that $a=\left(\operatorname{HomExtEval}\left(x, \mathbb{F}_{\mathbb{Q}}\right)\right)(g)$.

Let us consider elements $x, a$ of $\mathbb{C}_{\mathrm{F}}$. Now we state the propositions:

(52) Suppose $a \neq 0_{\mathbb{C}_{\mathrm{F}}}$ and $a \in$ the carrier of $\mathbb{Q}[x]$. Then there exists an element $g$ of PolyRing $\left(\mathbb{F}_{\mathbb{Q}}\right)$ such that

(i) $g \notin \operatorname{AnnPoly}\left(x, \mathbb{F}_{\mathbb{Q}}\right)$, and

(ii) $a=\left(\operatorname{HomExtEval}\left(x, \mathbb{F}_{\mathbb{Q}}\right)\right)(g)$.

The theorem is a consequence of (51).

(53) Suppose $x$ is algebraic and $a \neq 0_{\mathbb{C}_{\mathrm{F}}}$ and $a \in$ the carrier of $\mathbb{Q}[x]$. Then there exist elements $f, g$ of $\operatorname{PolyRing}\left(\mathbb{F}_{\mathbb{Q}}\right)$ such that

(i) $\{f\}$-ideal $=\operatorname{AnnPoly}\left(x, \mathbb{F}_{\mathbb{Q}}\right)$, and

(ii) $g \notin \operatorname{AnnPoly}\left(x, \mathbb{F}_{\mathbb{Q}}\right)$, and

(iii) $a=\left(\operatorname{HomExtEval}\left(x, \mathbb{F}_{\mathbb{Q}}\right)\right)(g)$, and

(iv) $\{f\}$-ideal and $\{g\}$-ideal are co-prime.

The theorem is a consequence of (28), (3), (52), (32), (29), and (50).

(54) Suppose $x$ is algebraic and $a \neq 0_{\mathbb{C}_{\mathrm{F}}}$ and $a \in$ the carrier of $\mathbb{Q}[x]$. Then there exists an element $b$ of $\mathbb{C}_{\mathrm{F}}$ such that

(i) $b \in$ the carrier of $\mathbb{Q}[x]$, and

(ii) $a \cdot b=1_{\mathbb{C}_{\mathrm{F}}}$.

The theorem is a consequence of (53), (3), (14), (15), and (20).

(55) Let us consider an element $x$ of $\mathbb{C}_{\mathrm{F}}$. If $x$ is algebraic, then $\mathbb{Q}[x]$ is a field. The theorem is a consequence of (54), (41), and (42). 


\section{REFERENCES}

[1] Michael Francis Atiyah and Ian Grant Macdonald. Introduction to Commutative Algebra, volume 2. Addison-Wesley Reading, 1969.

[2] Jonathan Backer, Piotr Rudnicki, and Christoph Schwarzweller. Ring ideals. Formalized Mathematics, 9(3):565-582, 2001.

[3] Grzegorz Bancerek. The fundamental properties of natural numbers Formalized Mathematics, 1(1):41-46, 1990.

[4] Grzegorz Bancerek and Krzysztof Hryniewiecki. Segments of natural numbers and finite sequences. Formalized Mathematics, 1(1):107-114, 1990.

[5] Czesław Byliński. Functions and their basic properties Formalized Mathematics, 1(1): 55-65, 1990.

[6] Eugeniusz Kusak, Wojciech Leończuk, and Michał Muzalewski. Abelian groups, fields and vector spaces. Formalized Mathematics, 1(2):335-342, 1990.

[7] Hideyuki Matsumura. Commutative Ring Theory. Cambridge University Press, 2nd edition, 1989. Cambridge Studies in Advanced Mathematics.

[8] Robert Milewski. The ring of polynomials Formalized Mathematics, 9(2):339-346, 2001.

[9] Robert Milewski. The evaluation of polynomials Formalized Mathematics, 9(2):391-395, 2001.

[10] Masayoshi Nagata. Theory of Commutative Fields, volume 125. American Mathematical Society, 1985. Translations of Mathematical Monographs.

[11] Andrzej Trybulec. Binary operations applied to functions Formalized Mathematics, 1 (2):329-334, 1990.

[12] Wojciech A. Trybulec. Non-contiguous substrings and one-to-one finite sequences Formalized Mathematics, 1(3):569-573, 1990.

[13] Wojciech A. Trybulec. Vectors in real linear space. Formalized Mathematics, 1(2):291-296, 1990.

[14] Oscar Zariski and Pierre Samuel. Commutative Algebra I. Springer, 2nd edition, 1975.

Received December 15, 2016 